\title{
Los fashion films como contenido particular del marketing de moda: un análisis de su naturaleza en el contexto de los mensajes híbridos*
}

\author{
Leslie Kam** \\ Kelly Robledo-Dioses*** \\ Tomás Atarama-Rojas *** \\ Recibido: 2018-10-02. Enviado a pares: 2018-10-15. \\ Aprobado por pares: 2018-11-29. Aceptado: 2018-12-15 \\ https://doi.org/10.22395/angr.v17n34a10
}

\begin{abstract}
Resumen
Las marcas deben aportar contenidos de valor que puedan atraer la atención de sus públicos para generar resultados positivos con respecto a sus objetivos de comunicación. Esto es mucho más relevante en el sector de la moda, en donde la construcción de valor simbólico -más que la exclusividad del producto- es imprescindible para relacionarse emocionalmente con un consumidor de carácter aspiracional. En la actualidad, la publicidad tradicional, paradigma del modelo push, deja de ser eficiente para alcanzar a los públicos. Ante esto, han tomado especial relevancia los mensajes híbridos y, en particular, el fashion film como un tipo de contenido audiovisual propio del marketing de moda. En esta investigación partimos de una revisión bibliográfica exhaustiva de, por un lado, product placement como un tipo de publicidad y, por otro lado, de content marketing, advertainment y branded entertainment como tipos de branded content para identificar los conceptos que se aproximan a los fashion films. Así, tras un análisis de los diferentes mensajes híbridos que podrían acoger la categoría del fashion film, resaltan características que llevan a la conclusión de que el objeto de estudio responde no solamente al advertainment, sino también al branded entertainment, aunque, debido a las particularidades del sector de la moda, en este último es necesario admitir la presencia irreparable de los productos en la historia, siempre y cuando estos no formen parte de la narrativa al ser expuestos de manera protagónica, sino que se establezcan como objetos de embellecimiento de los personajes.

Palabras clave: fashion film; marketing; branded content; branded entertainment; advertainment; moda; película; entretenimiento.
\end{abstract}

El presente artículo es resultado de investigación del desarrollo de una amplia línea de investigación sobre fashion films en la Facultad de Comunicación de la Universidad de Piura.

* Licenciada en Comunicación por la Universidad de Piura (Perú), actualmente se desempeña como profesora investigadora de la Facultad de Comunicación de la Universidad de Piura (Perú) donde imparte las asignaturas Fundamentos de la Planificación Estratégica, Marketing Digital, Proyectos de Comunicaciones de Marketing, Comportamiento del Consumidor e Investigación en Comunicación 1 y 2, su investigación se centra en el fashion marketing, branded content y branding. leslie. kam.03@gmail.com. Orcid: https://orcid.org/0000-0001-9777-8481

... Licenciada en Comunicación por la Universidad de Piura (Perú), profesora investigadora de la Facultad de Comunicación de la Universidad de Piura (Perú) donde imparte las asignaturas Comunicación Escrita 1, Géneros y Estilos Informativos e Investigación en Comunicación 1 y 2, su investigación se centra en el periodismo, la comunicación transmedia y el storytelling corporativo. kelly.Robledo@udep.pe. Orcid: https://orcid.org/0000-0003-1409-3773

"*** Máster en Creación de Guiones Audiovisuales por la Universidad Internacional de La Rioja (España) y en Comunicación por la Universidad de los Andes (Chile) y licenciado en Comunicación por la Universidad de Piura (Perú), se desempeña como profesor investigador de la Facultad de Comunicación de la Universidad de Piura (Perú) donde imparte las asignaturas Comunicación Narrativa y Storytelling, y Fundamentos de Guion, su investigación está centrada en el guion cinematográfico y en el uso del storytelling en el mundo comercial y corporativo. tomas.atarama@udep.pe. Orcid: https://orcid.org/0000-0002-4430-3391 


\title{
Fashion Films as a Particular Content of Fashion Marketing: An Analysis of its Nature in the Context of Hybrid Messages
}

\begin{abstract}
Brands must provide valuable content that can attract attention of their audiences to generate positive results regarding their communication objectives. This becomes much more relevant in the fashion industry, where the construction of symbolic value ---even more so than the product's exclusivity--- is essential to relate oneself emotionally with a consumer of aspirational nature. Currently, traditional advertising, which is a paradigm of the push model, is no longer efficient to reach the public. Given this, hybrid messages have become particularly relevant, and specially the fashion film as a kind of audiovisual content that is typical of fashion marketing. In order to identify the concepts that draw near to fashion films, this research began with an exhaustive literature review of, on the one hand, product placement as a type of advertising and, on the other hand, of content marketing, advertainment and branded entertainment as types of branded content. Thus, after an analysis of different hybrid messages that could host the fashion film category, features that lead to the conclusion that the subject matter responds not only to advertainment, but also to branded entertainment. Although, given the particularities of the fashion industry, within the latter it is necessary to admit the irreparable presence of products in history, as long as they do not take part in the narrative when they are exposed in a leading way, but rather establish themselves as objects of embellishment of the characters.
\end{abstract}

Keywords: fashion film; marketing; branded content; branded entertainment; advertainment; fashion; movie; entertainment.

\section{Os fashion films como conteúdo particular do marketing de moda: uma análise de sua natureza no contexto das mensagens híbridas}

\section{Resumo}

As marcas devem oferecer conteúdos de valor para que possam atrair a atenção de seus públicos para gerar resultados positivos com respeito a seus objetivos de comunicação. Isso é muito mais relevante no setor da moda, onde a construção de valor simbólico - mais do que a exclusividade do produto - é imprescindível para se relacionar emocionalmente com um consumidor de caráter aspiracional. $\mathrm{Na}$ atualidade, a publicidade tradicional, paradigma do modelo push, deixa de ser eficiente para alcançar os públicos. Em face disso, as mensagens híbridas e, em particular, o fashion film, ganharam especial relevância como um tipo de conteúdo audiovisual próprio do marketing de moda. Nesta pesquisa partimos de uma revisão bibliográfica exaustiva de, por um lado, product placement como um tipo de publicidade e, por outro lado, de content marketing, advertainment e branded entertainment como tipos de branded content para identificar os conceitos que se aproximam aos fashion films. Assim, depois de uma análise das diferentes mensagens híbridas que poderiam acolher a categoria do fashion film, ressaltam as características que levam à conclusão de que o objeto de estudo responde não somente ao advertainment, mas também ao branded entertainment, embora, devido às particularidades do setor da moda, neste último é necessário admitir a presença irreparável dos produtos na história, sempre e quando eles não fizerem parte da narrativa ao serem expostos de maneira protagonizada, mas que se estabeleçam como objetos de embelezamento dos personagens.

Palavras-chave: fashion film; marketing; branded content; branded entertainment; advertainment; moda; filme; entretenimento. 


\section{Introducción}

Actualmente, el consumidor es el núcleo de cualquier estrategia de comunicación. Ya no es posible sostener el valor de una marca en la economía actual en base a información que se brinda de manera unilateral, o se genera vinculación (emocional y psicológica) con el consumidor o la sentencia de este exigente público será la indiferencia o el olvido. En cambio, a través de la generación de engagement y la formación de una comunidad en torno a unos valores propuestos por la marca, el consumidor se sumará a los fines comerciales y será el principal defensor de la marca en un ecosistema mediático de múltiples entradas.

Como explican De Aguilera-Moyano, Baños-Gonzáles y Ramírez-Perdiguero (2015, p. 520), "estamos ante un nuevo escenario de marketing en el que el engagement del consumidor es la fuerza motriz que está detrás de su comportamiento y de la toma de decisiones". Por esto es necesario trabajar contenidos que favorezcan la relación con el público. Esto exige contenidos creativos que no sean intrusivos, sino que por su relevancia sean buscados por el propio consumidor, en otras palabras, se requiere superar el marketing push (que se encuentra en declive), para apostar por estrategias pull (De Aguilera-Moyano et al., 2015). Para Del Pino-Romero y Castelló-Martínez (2015, p. 106):

Es importante resaltar la evidencia de cómo las estrategias pull, surgidas a raíz del fin de las audiencias cautivas, son las únicas que realmente dan sentido y explican el nuevo panorama audiovisual y publicitario, en donde el consumidor -en tanto que individuo y usuario-, tiene la última palabra.

En este nuevo contexto, queda claro que la publicidad tradicional, paradigma del modelo push, deja de ser eficiente para alcanzar al público. Así, toman protagonismo los mensajes híbridos (De Aguilera, Baños y Ramírez, 2016). Estos contenidos resaltan por ser una propuesta de valor para el público bajo un formato no comercial. En este sentido, "los mensajes híbridos combinan, de alguna forma, el mensaje comercial de una marca con un contenido no comercial capaz de proporcionar una experiencia positiva" (De Aguilera-Moyano et al., 2015, p. 522). Así, este tipo de mensaje responde al nuevo modelo de comunicación que exige el público actualmente, donde lo esencial no es imponer o repetir, sino atraer y sugerir (Ramos, 2006).

Dentro de los denominados mensajes híbridos, se puede hablar del product placement como uno de los modelos precursores y, ya en la dinámica actual, del branded content, content marketing, branded entertainment, advertainment, advergaming, custom publishing, entre otros. Como destacan De Aguilera-Moyano et al. (2015):

La ventaja fundamental de este tipo de mensajes sobre la marca está en que el espectador los recibe con mayor atención y receptividad, no atribuyendo su presencia a una acción de comunicación del anunciante sino a las características del contenido de entretenimiento que ha decidido consumir (p. 523).

Ante esto, existe consenso en la conveniencia de apostar por estos nuevos modos de comunicar. De hecho, el entorno académico y profesional ha intentado delimitar con exactitud el campo, las características y la naturaleza de cada uno de estos mensajes (Ramos- 
Serrano y Macías-Muñoz, 2016). Aun así, entre el debate y la ambigüedad nominal, en los últimos años han adquirido especial relevancia, popularidad e influencia en la industria de la moda los denominados fashion films (Del Pino-Romero y Castelló-Martínez, 2015) y aunque nuestro interés se centra en delimitar adecuadamente el concepto y las virtudes de los fashion films, se ha considerado metodológicamente relevante aclarar los alcances de la pluralidad de estos tipos de contenidos para reconocer si se puede considerar a esta nueva forma de comunicación del sector de la moda como formato de alguno de estos mensajes híbridos ya tipificados, o si, por sus características particulares, puede solicitar con autoridad el reconocimiento de ciudadanía individual en este campo.

Por esta razón, en esta investigación partimos de una revisión bibliográfica exhaustiva para dar luces respecto a los conceptos que sirven como sustento o que se aproximan a los fashion films: publicidad, product placement, branded content, content marketing, branded entertainment $\mathrm{y}$ advertainment. Partimos de la premisa de que el lenguaje siempre refiere a una realidad y que la diferente denominación obedece a la existencia de características particulares, ya sea a nivel esencial, estructural o funcional. Nuestro primer esfuerzo apunta a determinar los elementos propios de cada tipo de mensaje para reclamar identidad propia.

Finalmente, nos aproximamos a los fashion films para entender, primero, las particularidades de la moda como objeto de comunicación y explorar los alcances de su naturaleza en la composición de los mensajes de marketing y, segundo, para proponer su carácter de mensaje específico del sector de la moda. Al profundizar en el concepto del fashion film, delimitando sus alcances, se podrá determinar si se le puede considerar un tipo de mensaje híbrido propio del sector de la moda.

\section{Metodología}

La investigación tiene carácter fundamentalmente teórico, porque se trata de una revisión conceptual y taxonómica de la terminología utilizada en la industria y la investigación académica relacionada con el marketing y los mensajes híbridos aplicados al campo de la moda. Por esto, la metodología utilizada es la revisión bibliográfica de las publicaciones académicas atinentes los mensajes híbridos. Las palabras clave para realizar la búsqueda en las bases de datos académicas fueron las siguientes: publicidad, product placement, branded content, content marketing, branded entertainment, advertainment, y fashion films. La búsqueda se realizó en las bases de datos de Dialnet, Scielo y Scopus, y en el repositorio académico de Google Scholar. El periodo de revisión bibliográfica se ubica entre los años 1994 y 2017. En su mayoría, las fuentes revisadas han sido producidas en los últimos cinco años. A partir de esta revisión de la literatura, se ha realizado la esquematización de cada uno de los conceptos para ordenar y diferenciar los aportes de cada uno en el área de las comunicaciones de marketing. En el apartado de resultados, primero se presenta la clasificación según las relaciones y diferencias de cada uno de los mensajes. Después, se muestra la delimitación conceptual y práctica del fashion film. Así, luego de analizar toda la información, se determina, finalmente, a qué mensaje híbrido corresponde el fashion film. 


\section{Resultados}

\section{Publicidad}

Entre las diversas propuestas de definiciones de publicidad generadas a partir de los años noventa, encontramos a autores como Wells, Burnett y Moriarty (1996, p. 12), quienes sostienen que la publicidad es "comunicación impersonal pagada por un anunciante identificado que usa los medios de comunicación con el fin de persuadir a una audiencia, o influir en ella". Asimismo, Ortega (1997, p. 22) defiende que la publicidad es el "proceso de comunicación de carácter impersonal y controlado que, a través de medios masivos, pretende dar a conocer un producto, servicio, idea o institución con objeto de informar o de influir en su compra o aceptación". En estas definiciones se observan factores en común, como el uso de medios masivos pagados por un anunciante en concreto. Además, se evidencia que el fin de la publicidad no es exclusivamente motivar la compra, sino conseguir que el público al que esta se dirige responda de manera favorable al contenido del mensaje, dado que las campañas de organizaciones sin fines lucrativos no suelen relacionarse con objetivos de venta. De este modo, la publicidad engloba una serie de contenidos que pueden trascender los límites comerciales, teniendo además como fin comunicar ideas con el propósito de influir en su aceptación.

Por otro lado, existen autores que resaltan que la publicidad es una estrategia promocional, y que su mayor reto es influir en el comportamiento de compra. Kumar y Raju (2013, p. 37) la definen como "una herramienta importante en la creación de conocimiento del producto en la mente del consumidor potencial para tomar una decisión de compra eventual". De este modo, aseguran los autores, la misión primaria del publicista es incidir en el conocimiento del producto para que el consumidor lo tenga presente durante el proceso de decisión de compra. En esta línea, de acuerdo a Suárez (2012, p. 32), "la publicidad resulta una comunicación donde el mensaje es trabajado en base a la originalidad, pero atendiendo específicamente a su función final: persuadir acerca de la conveniencia en la elección de una marca". De este modo, la publicidad se dedica a desarrollar mensajes persuasivos con un fin comercial. Para lograrlo, la comunicación se basa en una serie de argumentos para convencer al público de adquirir dichos bienes. Así, como se ha venido manifestando en las definiciones anteriores, el carácter persuasivo es connatural en la publicidad.

En un principio, la persuasión se basaba exclusivamente en los atributos de los productos, como su calidad, sus funciones y su utilidad; hoy se habla de una nueva publicidad, en donde las marcas intentan crear aquellos contenidos, ideas, experiencias o emociones para el disfrute del receptor de la comunicación (Del Río y Kaufmann, 2014). De esta manera, la publicidad se aleja cada vez más de la descripción física del producto para convertirse en un punto de acceso al universo simbólico de las marcas. Así, el producto se presenta de una forma cada vez más implícita, y la marca se inmiscuye y forma parte del relato o la situación para lograr mayor familiaridad a la hora de la comunicación del bien. En este sentido, "se ofrecen bienes cuyas ventajas competitivas suelen ser muy sutiles en el aspecto material pero que difieren en su construcción simbólica" (Suárez, 2012, p. 31). 
Esto se refiere, en el fondo, a la denominada "publicidad de la significación", término acuñado por Antonio Caro (1994), quien la diferencia de la llamada "publicidad referencial". La publicidad referencial se concentra en el producto y simplemente se limita a darlo a conocer, enfatizando su utilidad para el consumidor. De tal modo que se trata de una publicidad básicamente informativa, que intenta persuadir, mediante recursos retóricos, sobre la superioridad del producto anunciado frente a la competencia. Por el contrario, la publicidad de la significación se centra en la marca y en atribuirle el significado más pertinente para atraer al consumidor. De esta manera, estamos hablando de una "publicidad que ya no tiene como referencia un producto o una entidad preexistente que hay que dar básicamente a conocer, sino una marca (un signo, en definitiva) a la que hay que dotar de una significación específica" (Caro, 1994, p. 120).

Dado que este último tipo de publicidad se centra en atribuir significado a las marcas, es preciso especificar entonces que la marca es un aparato semiótico que construye, concede y difunde significados en torno a un producto, bien o servicio y que, además, está formado por el conjunto de los discursos que mantienen entre sí todos los sujetos que participan en su origen (Semprini, 1995). Dicho esto, la publicidad se encarga, precisamente, de construir marcas (Caro, 1994). En este sentido, como sostiene Eguizábal (2007, p. 129), "el proceso publicitario se convierte en un proceso semiótico por el cual no son ya los objetos los que se revisten de significado, son los conceptos los que se procuran una apariencia, una materialidad".-

En definitiva, concordamos con que la publicidad lleva a cabo una función constructiva de marcas dotándolas de significación para envolverlas en un mundo imaginario y, por ende, conferirles valor de consumo. En este sentido, se entiende que la publicidad participa de la producción semiótica de las marcas, construyendo universos simbólicos alrededor de esta.

\section{Product placement}

Como primer escalón dentro del conjunto denominado como mensajes híbridos, tenemos al product placement, o emplazamiento de producto, que surge como una herramienta alternativa a la publicidad tradicional recurriendo a diferentes medios y soportes comunicativos (García, Sánchez y Torrano, 2014), y con un fin y control de los mensajes también distintos (Guerrero, 2015).

El nacimiento del product placement abre una nueva puerta para la publicidad de un producto o marca: que este se inserte en un contenido ajeno que será consumido por el público'. Esto, como señalan Cordero (2015ª; 2015b) y Pérez, Navarrete y Gómez (2014), se presencia principalmente en el discurso audiovisual, se usa, entonces, en programas

\footnotetext{
"Cuando se emplaza un producto en la acción de una película, lo que se está haciendo en realidad es entrelazar un mensaje comercial o una acción comunicativa de índole comercial, con otro mensaje cuyo objetivo comunicativo es diferente: el entretenimiento" (Rodríguez y Baños, 2013, p. 149).
} 
y series de televisión, telenovelas, cine, videos musicales, videojuegos, entre otros, sin descartar la actuación análoga en términos equivalentes en el medio radio².

En la academia se ha escrito mucho sobre product placement. A continuación, presentamos una definición que consideramos la más completa:

Una técnica de comunicación comercial que consiste en introducir la publicidad de un producto, de un servicio o de una marca dentro del escenario argumental en el que se desarrolla la acción de una película o de un producto audiovisual, llegando al punto de que el producto, el servicio o la marca se acaba mezclando con los personajes y la trama (Cordero, 2015b, p. 42).

La inserción del producto, servicio o logo, dentro de la narrativa de la pieza, consiste en exhibirlo, mencionarlo o ser utilizado por los actores, con una intención y una finalidad económica, a cambio de unas tasas pagadas al productor o de una colaboración en la promoción de esas obras (Cordero, 2015b; Méndiz, 2000; Morgado, 2008). Por ello, explica Morgado (2008, p. 5), la clave está en "lograr insertarlo de una forma sublime, sutil, incluso transgresora, porque eso es lo que vende, y eso es lo que nos va a hacer captar la atención de todos aquellos clientes potenciales" que se encuentran dispersos en diversas actividades y consumen diversos medios.

Actualmente el product placement ha crecido enormemente y cuenta con unas expectativas de crecimiento muy positivas para el futuro (Redondo y Bernal, 2015). Para Morgado (2008), es un fenómeno publicitario en expansión. Ya no se trata solo de colocar el producto a modo de atrezzo, sino que se gestiona desde los despachos, de manera que el producto insertado en la ficción tiene un lugar estratégico. Aunque con esto último se debe ser muy cuidadoso, pues cuando el protagonismo del producto es más marcado que el de los personajes, el público se da cuenta de que la inserción es exagerada, y se puede ocasionar el efecto contrario de la compra (Morgado, 2008; Rodríguez, 2012), y será inevitable entonces que el consumidor lo compare con las herramientas publicitarias más tradicionales (Khalbous, Vianelli, Domanski, Dianoux y Maazoul, 2013).

Como destaca Rodríguez (2012, p. 25), la intención del product placement es la de "integrar el mensaje comercial a la trama", lo que convierte a los productos, servicios y marcas en elementos constitutivos del universo diegético de las ficciones. De esta manera, "en el mensaje que recibe el público, el objetivo comercial que persigue la comunicación del anunciante se entrelaza, de manera inevitable, con el significado específico de la historia que se narra en la película" (Rodríguez y Baños, 2013, p. 149).

A propósito de esto último, cabe destacar dos categorías generales de product placement en las que varios autores (García et al., 2014; Guerrero, 2015; Rodríguez, 2012) han coincidido: 1) prominente: es una mención de producto o marca de alta intensidad, en la que el producto u otro identificador de la marca se hace muy visible por su tamaño o posición en la pantalla, y en la que los actores la usan o se refieren a ella y sus bondades; 2) sutil: se trata de una mención de baja intensidad; no se presenta de manera destacada

Comenzó su uso en el cine y se prevé un gran desarrollo a futuro en los videojuegos (Cordero, 2015b). 
por la pequeña dimensión del producto, por un tiempo de exposición menor, por ocupar un lugar fuera del campo principal de enfoque visual y porque no hay una referencia verbal hacia ellos.

En el siguiente punto abordaremos otro tipo de mensaje híbrido, el branded content, que, como señalan Formoso, Sanjuán y Martínez (2016), suele causar confusiones con el product placement.

\section{Branded content}

Existe una gran distinción entre branded content y product placement. Formoso et al. (2016) señalan que, si bien ambas modalidades publicitarias no interrumpen el momento de ocio del espectador, pues se integran al contenido ficcional, la principal diferencia se encuentra en la autoría del contenido. El creador/productor del contenido para el caso del product placement es un tercero, mientras que en el branded content es el mismo anunciante. Pero, además de la autoría, la intencionalidad en la creación del contenido es otra diferencia (Díaz y García, 2016). El branded content se trata de la producción y distribución de contenidos de la marca cuya finalidad va más allá de lo meramente publicitario, pues "los anunciantes buscan entretener, emocionar, divertir o sorprender al espectador (...) generando así un vínculo emocional con él" (Formoso et al., 2016, p. 17).

Otra de las diferencias es que "el product placement no es contenido buscado por el usuario, mientras que el branded content sí lo es" (Formoso et al., 2016, p. 17). Con este último se trata de implementar una estrategia publicitaria pull y no push. De esta forma, el contenido creado por el branded content debe ser tan bueno que logre "transmitir un mensaje de marca, de manera más o menos explícita" (De Aguilera et al., 2016, p. 41), que despierte el interés del consumidor.

Luego de lo visto, no es difícil definir al branded content como "la creación por la marca de sus propios contenidos" (De Aguilera et al., 2016, p. 39). En esta línea, Del Pino-Romero y Castelló-Martínez (2015, p. 112) manifiestan que el branded content consiste en "un espacio de información y/o entretenimiento con contenidos audiovisuales cuya producción ha sido desarrollada o participada por la marca". Los autores explican que este tipo de mensajes "están al servicio de la marca desde el momento de su concepción, aunque esta no siempre tenga presencia explícita en ellos" (Del Pino-Romero y Castelló-Martínez, 2015, p. 12).

Segarra-Saavedra e Hidalgo-Marí (2013, p. 1) detallan que el branded content "convierte a la marca en sujeto activo de la historia, en cuanto el contenido y la evolución narrativa gira en torno a la filosofía de la marca". De esta manera, este tipo de contenido utiliza el poder de las historias para conectar con el consumidor, generando movilización y deseo de forma indirecta y sutil, con lo cual la marca entreteje su presencia con el discurrir de un mensaje a través de un hilo emocional (Castelló-Martínez y Del Pino-Romero, 2014; Del Pino-Romero y Castelló-Martínez, 2015; Segarra-Saavedra, Hidalgo-Marí y Tur-Viñes, 2016). 
El branded content se ha popularizado como formato alternativo de comunicación publicitaria (Castelló-Martínez y Del Pino-Romero, 2014). Se trata de una estrategia en la que el anunciante se convierte en productor de contenidos que integran a los valores de una marca (Pineda, 2009; Segarra-Saavedra, 2016). Aguado (2008) explica que estos contenidos pueden ser de entretenimiento, información o educación, ya sea a través de las pantallas compartidas, patrocinio, de modalidades como el advertainment y el licensing o, incluso, mediante el emplazamiento de producto. Se ve entonces que "abarca técnicas y formatos muy dispares. (...) No solo es un enfoque de comunicación de marca que se utiliza únicamente en medios de carácter audiovisual, aunque estos sean los más conocidos y populares" (Caballero, 2014, p. 9).

De este modo, es posible sostener que el branded content es una forma de comunicación que abarca todo contenido de carácter informativo, educativo y de entretenimiento creado por la propia marca, con la finalidad de comunicar sus valores mediante de un formato lúdico y atractivo para el público (Ramos-Serrano y Macías-Muñoz, 2016). Así, como manifiesta Martínez (2011, p. 553), el "Granded content es una categoría superior que engloba todos los contenidos producidos para una marca". Precisamente, el content marketing, branded entertainment y advertainment son mensajes considerados tipos de branded content.

Después de este acercamiento al branded content, a continuación, trabajaremos tres modos en los que este se desarrolla: el content marketing, el branded entertainment y el advertainment.

\section{Content marketing}

Como parte del branded content, el content marketing es una técnica que busca crear y distribuir contenido relevante y valioso para atraer, adquirir e involucrar a un público objetivo claramente definido y comprendido (Patrutiu, 2015; Pulizzi, 2012). Sin embargo, tiene una particularidad que lo diferencia de sus pares, el branded entertainment y el advertainment.

Diversos autores coinciden en que el content marketing tiene como principal fin el educar al consumidor, para lo cual se centra en generar contenido de carácter informativo y educativo (De Aguilera et al., 2016; Content Marketing Institute, s. f.; Rancati y Gordini, 2014; Hallahan, 2014; Lieb, 2011; Patrutiu, 2015; Rahim y Clemens, 2012). De Aguilera et al. (2016, p. 43) nos ofrecen una definición completa de content marketing:

Técnica de marketing que consiste en la creación y distribución, por parte de una marca, de contenido relevante, valioso y convincente, a través de medios propios y/o ganados, para atraer, adquirir, educar y engage a clientes y prospectos; el contenido puede ser informativo y/o educativo y, si bien no realiza ninguna propuesta de venta o llamada a la acción, tiene como objetivo el promover una acción beneficiosa para la marca por parte del cliente y/o prospecto.

Como explica Patrutiu (2015, p. 116), "el principal papel que desempeña el content marketing es informar y educar al público para desarrollar una relación privilegiada con él y para determinar la lealtad a la marca". Para conseguirlo, se basa en la creación y distri- 
bución de "valiosas piezas de información que enriquecen a la comunidad y posicionan a la marca como líder en su campo", este contenido, además de que puede compartirse inmediatamente, se focaliza en ayudar a los clientes a descubrir (por sí mismos) que el producto o servicio es el que resuelve su necesidad (De Aguilera et al., 2016, p. 43).

"El propósito de esta información no es dar a conocer las virtudes de los propios productos o servicios del anunciante, sino informar al público objetivo y potencial acerca de los principales problemas de la industria, involucrando quizá los productos propios" (Patrutiu, 2015, p. 116). En otras palabras, con la información que se le otorga al cliente a través del content marketing, se le está diciendo que su compra es inteligente. "La esencia de esta estrategia de contenido es la creencia de que si nosotros, como empresas, entregamos información valiosa y constante a los compradores, finalmente nos premian con su compra y lealtad" (Content Marketing Institute, 2015, citado por Patrutiu, 2015, p. 116).

Como vemos, ganar la fidelidad del consumidor resulta básico. "El content marketing trata de una marca, de lo que la hace única, y de su contexto; y puede utilizarse tanto como herramienta para generar ventas, como herramienta de largo plazo para construir confianza" (De Aguilera et al., 2016, p. 45). Esto quiere decir que "los consumidores intercambian lealtad por conocimiento, sobre todo si las marcas se consolidan como verdaderas expertas en su campo" (De Aguilera et al., 2016, p. 45).

Para cerrar este punto, merece destacar otra oportunidad que el content marketing le ofrece al anunciante. Además de ganar la lealtad del consumidor, el uso de esta técnica podría hacer que este se consolide como experto en su campo (De Aguilera et al., 2016). Se entiende pues que, al dominar y compartir información valiosa de su ámbito, el anunciante se encuentra a la vanguardia del sector y destaca entre sus pares.

\section{Branded entertainment}

El branded entertainment se define como una categoría del branded content, con la particularidad de que apunta a generar engagement o vinculación emocional con el público a través del entretenimiento. La delimitación del branded entertainment resulta ambigua, en cuanto el entretenimiento tiene una acepción amplia y una acepción estricta. Si tomamos la definición amplia de entretenimiento, sería branded entertainment cualquier contenido generado por las marcas que pueda captar la atención del público para distraerlo o romper con su rutina, contenido destinado a ser consumido en tiempos de ocio, ya que "los contenidos, especialmente los audiovisuales, se han convertido en la piedra angular para generar interés y atención en los usuarios" (Del Pino-Romero y Castelló-Martínez, 2015, p. 106).

Sin embargo, lo más acertado es entender el branded entertainment desde una acepción estricta de entretenimiento, que se corresponde con la creación de ficciones, con la estructura de un relato, que permitan al público entrar en mundos posibles que exploren nuevos rincones del alma humana desde las historias. Es importante señalar que esto se puede dar en un Reality Show o en una película del género fantástico, lo importante no es el grado de realidad presente en el relato, sino la creación de un universo que permite 
recrear la realidad con un sentido. Por esto, podemos afirmar que branded entertainment se distingue del content marketing en cuanto aporta contenidos de entretenimiento vinculados a la creación o recreación de historias, mientras que content marketing está relacionado con contenido informativo o educativo. El branded entertainment "es la forma que adopta el branded content cuando es entretenimiento, por oposición al content marketing, que es informativo/ educativo" (De Aguilera et al., 2016, p. 46).

En este sentido, el branded entertainment es la técnica que le permite a la marca crear verdaderas historias donde puede constituir su identidad y personalidad, así se puede controlar perfectamente la integración de los valores de la marca en el contenido (Lehu, 2007). Esto resulta especialmente relevante en un contexto donde el público exige contenido que le aporte algún valor. Se requiere:

Contenido relevante, por útil o por entretenido, que atraiga y mantenga la atención de sus públicos objetivos, sorteando su resistencia natural a la publicidad; bajo la premisa de que una experiencia positiva con el contenido generará sentimientos positivos hacia la marca que lo ha hecho posible (Duncan, 2012, citado en De Aguilera et al., 2016, p. 39).

Entonces, podemos enunciar algunas particularidades del branded entertainment. Para comenzar, su materia no es un producto o servicio concreto de la marca, sino la historia que transmite los valores de la marca; luego, entendemos que no se manifiesta de modo explícito la marca a través de sus productos, porque su objetivo de comunicación está a un nivel más abstracto y de generación de valor a partir del mismo contenido y, finalmente, admite diferentes niveles de construcción de la historia que se puede manifestar a través de diversos modos (desde una canción hasta un largometraje).

Es importante señalar que no existe consenso respecto a la idea de que el branded entertainment tenga que estar libre de una manifestación explícita del producto de la marca, pero los autores de esta investigación sostienen que esta es la principal diferencia con el advertainment. Por ello, reservamos al branded entertainment la característica de no presentar el producto de la marca. En la línea de los autores que consideran que el branded entertainment puede o no tener presencia de marca, se encuentran De Aguilera et al. (2016), quienes resaltan que cuando esta no existe, es importante otros esfuerzos de comunicación para vincular el contenido a la marca:

Incluso la pieza de entertainment puede llegar a estar totalmente desprovista de la presencia de la marca o de sus productos limitándose a reflejar la personalidad de la marca; en este caso, lo más probable es tener que recurrir a otras acciones de comunicación, notoriamente en medios propios y ganados, pero ocasionalmente también pagados, que garanticen el vínculo entre la pieza de entretenimiento y la marca (p. 48).

Cabe mencionar que cuando los autores citados en el presente estudio afirman que el branded entertainment "no admite presencia de la marca", están utilizando el término de modo análogo, dado que la marca, como motor semiótico, debe ser impregnada en el mundo imaginario que pretende construir para proyectar sus valores. Así, es correcto 
sostener que el branded entertainment sí admite la marca en sus contenidos, pero no precisamente el producto o el logo.

Una idea importante que resalta en el branded entertainment es justamente su valor independiente como pieza de entretenimiento. Esto significa que el branded entertainment debe poder funcionar como contenido de ficción o de entretenimiento para entrar a competir por un espacio en los canales de distribución de la industria. Esto está relacionado con la capacidad de generar rentabilidad en el producto, ya que:

Una pieza producida por una marca, actuando esta como productora, puede cumplir el papel de cualquier otra pieza de entretenimiento en la parrilla de una cadena, a coste cero por esta, lo que se traduce en una mejora de la cuenta de resultados, de la misma manera que lo haría un ingreso (De Aguilera-Moyano et al., 2015, p. 525).

Finalmente, retomando lo señalado en relación al branded entertainment podríamos definirlo como contenido de entretenimiento (no como publicidad referencial) creado por una marca, con un alto nivel de calidad para que pueda ser preferido por el público frente a otros contenidos no producidos por marcas. Este contenido debe acercar los valores de la marca al público y debe ser capaz de generar comunidad que facilite después la comunicación ya propiamente comercial con ese público. En esta línea, un antecedente del branded entertainment serían justamente las soap operas de la primera mitad del siglo pasado (Lehu, 2007) en las que "algunos fabricantes de productos de gran consumo, entre ellos los fabricantes de jabón (soap en inglés), invirtieron en la producción de programas de radio como mejor manera de alcanzar a sus públicos objetivos" (De Aguilera-Moyano et al., 2015, p. 524).

\section{Advertainment}

Varios autores (De Aguilera et al., 2016; Díaz y García, 2016; Garrido y Fernández, 2014; Ramos, 2006) consideran que el advertainment es un modo distinto de denominar al branded entertainment. Para este grupo de autores, ambos términos serían funcionalmente sinónimos. También, encontramos autores como Del Pino-Romero y Castelló-Martínez (2015), que sostienen que la categoría más amplia sería justamente la de advertainment, por su tradición y origen, y que en ella se podrían incluir las nuevas formas de mensajes híbridos. Sin embargo, sostenemos que advertainment y branded entertainment no son sinónimos, sino que cada uno responde a una naturaleza distinta.

De acuerdo a Martínez (2011, p. 551), el advertainment es un "síntoma de cambio estructural en la relación de las marcas con sus públicos" en donde la exaltación de los beneficios diferenciales de los productos es superada por el consumo simbólico. Efectivamente, este tipo de contenido se presenta como publicidad revestida de entretenimiento, pero continúa siendo publicidad en el fondo, por lo que el producto debe aparecer obligatoriamente en alguna parte del contenido, ya sea incorporada en el desarrollo narrativo del entretenimiento, o simplemente como un elemento que puede acompañar a la historia. En esta 
misma línea, los estudios de Pineda, Pérez y Hernández-Santaolalla (2013, p. 85) indican que la integración de valores de marca coexiste con la presencia física de productos en la narración y que, debido a esto, este tipo de contenidos están sometidos a "una visión creativa donde sigue siendo necesario emplazar y dotar de gran visibilidad al producto; lo cual va en detrimento de a) la sutileza que se le presupone al branded content, y b) una relación entre marcas, personajes y contexto fílmico que sea natural y no estridente".

De este modo, el advertainment sería, como dicen Del Pino-Romero y Castelló-Martínez (2015, p. 109), "una simbiosis entre publicidad y contenidos de entretenimiento", es decir, el resultado de hibridar la publicidad con el entretenimiento, pero con cuidado de que se trate en el fondo de publicidad, aunque en la forma se materialice como entretenimiento. Así, podemos estar hablando de anuncios especialmente interesantes por su estética y su guion (Ramos, 2006), anuncios con un formato más amable con el consumidor, que busca entretenerlo, "evitando cualquier recurso tendente a interrumpirle o distraerle del disfrute de ese contenido" (Regueira, 2012, p. 112). Por esto, aunque la diferencia sea muy sutil, es importante diferenciar el concepto de advertainment del de branded entertainment porque el mensaje resulta distinto y los objetivos que se perfilan desde el punto de vista de la comunicación también son diferentes. Así, podemos considerar las siguientes diferencias:

\section{Nivel de aparición de la marca:}

- Branded entertainment: no aparece el producto, el servicio o el logo durante la historia, pero sí se evidencian los valores de la marca en el mundo imaginario presentado.

- Advertainment: aparecen los productos o servicios que se procura publicitar de manera protagónica en la historia.

\section{Objetivo de comunicación:}

- Branded entertainment: transmitir los valores de la marca y generar una comunidad en torno a propuestas de valor y relaciones significativas.

- Advertainment: generar posicionamiento, introducir o recordar un producto o servicio.

Como se puede apreciar, aunque ambas formas de branded content son contenidos de entretenimiento generados por la marca, es posible y recomendable diferenciarlos para propiciar un correcto desarrollo de ambas formas de comunicación y evitar que la confusión redunde en la falta de comprensión de la técnica que se puede desarrollar.

\section{Propuesta de delimitación conceptual y práctica: los fashion films}

\section{Las particularidades de las marcas de moda}

Diversos autores (Franck, 1990; Sánchez, 1999; Del Olmo, 2005; Kawamura, 2005) coinciden en que el uso de las prendas de vestir es una de las actividades más significativas entre 
todas aquellas que tienden a convertirse en moda. Esto se debe a que el valor de la vestimenta no radica en su uso básico - protección del clima y de la desnudez en público-, sino en su función social. Como explica Sánchez (1999), los individuos no solo se visten para ellos mismos, sino para los demás. Así, la moda tiene una doble vertiente: como un medio de integración y como un recurso para el embellecimiento. En este sentido, se sostiene que las personas quieren ser vistas de forma agradable y formar parte de un grupo; y es que la indumentaria, al configurarse como un soporte evidente, flexible y fácil de imitar, posibilita la manifestación explícita de la aceptación de una determinada sociedad. De esta manera, existe una tendencia a la uniformización, en la cual la ropa funciona como un indicador de pertenencia a un determinado grupo social, caracterizado por un estilo de vida definido (Martín, 2008). Dicho esto, en la moda las tendencias a la igualdad social, la diferenciación individual y la variedad se unifican (Simmel, 1976, citado por Herrero, 2004).

En este sentido, Martínez y Vázquez (2006) y Gómez, Calvo y Otero (2013) sostienen que los productos de moda satisfacen las necesidades sociales y psicológicas de las personas, y que, por esta razón, son muy emocionales a diferencia de otros tipos de bienes:

Los bienes de moda tienen en su ADN el componente emocio-aspiracional, es decir, la necesidad de satisfacer necesidades sociales y emocionales. Por ello, basar el marketing en un comportamiento estrictamente racional de los individuos es alejarse ostensiblemente de la realidad de las marcas de moda, de su demanda y de la forma en que pueden ser "vendidas" a través del marketing (Gómez et al., 2013, p. 29).

Por esta razón, las marcas de moda deben recurrir a argumentos emocionales que se acerquen más a los deseos del consumidor (Martín, 2008). Bajo este contexto, autores como Michaud (2015) resaltan que las marcas de moda pertenecientes al sector de lujo son las que más deben preocuparse por atraer de manera irracional al consumidor, utilizando símbolos referidos a nociones abstractas como la excelencia, la belleza o el poder. Como sostienen Kapferer y Valette-Florence (2016), esto se debe a que los precios altos de los productos de lujo no se justifican del todo por sus características funcionales porque, en realidad, las marcas de lujo se dedican a generar un valor que sobrepasa la satisfacción derivada de un producto de calidad superior, así, el precio proviene de las singularidades construidas por los intangibles, tales como la tradición, la historia, el país de origen, o el estilo de vida imaginario de las marcas.

De esta manera, es necesario que las marcas de moda de lujo acumulen un capital simbólico que no se limite a la exclusividad del producto, sino que se dediquen a construir un mundo simbólico, y forjar deseabilidad en el consumidor para que procure pertenecer a este. En este sentido, se entiende que el detonante de la elección de marcas de moda de lujo es la aspiración de pertenencia, por ello, como afirma Martín (2008, p. 110), estas "se construyen sobre mundos idílicos, monotonías glamurosas y personalidades aspiracionales". Ante este panorama, los fashion films siguen una estrategia de comunicación aspiracional propia del lujo, suscitando el deseo de formar parte del estilo de vida que las marcas de moda ofrecen. 


\section{Aproximación a los fashion films}

El fashion film se encuentra en el debate de dos puntos de vista: por un lado, se sostiene su índole meramente artística, propia de la cinematografía y, por otro lado, se defiende su exclusiva naturaleza publicitaria. En este escenario, Uhlirova (2013a, p. 120) manifiesta que esta discusión académica surge fácilmente debido a que "el fashion film parece aplicarse sin problemas a tipos más directos de publicidad y, al mismo tiempo, a trabajos que están evidentemente más dedicados a la expresión del autor que en la generación de beneficios promocionales para los productos o marcas".

En este sentido, el tipo de mensaje híbrido que responde inevitablemente a la perspectiva publicitaria del fashion film es el advertainment. En este primer contexto, diversos académicos ya han sustentado que el fashion film es una simbiosis entre entretenimiento y publicidad. Por ejemplo, Caerols y De la Horra (2015, p. 354), quienes afirman que "los fashion film no son exactamente anuncios, ni cortometrajes, ni vídeos artísticos o musicales, sino una mezcla de todos ellos, respaldados por grandes nombres de directores, fotógrafos y actores de cine, destinados muchas veces exclusivamente para la red, a convertirse en video viral". Otros autores que se suman de manera similar a esta posición son Del Pino-Romero E Castelló-Martínez (2015, p. 118), quienes observan una fusión de arte, cine, música, moda y publicidad en los fashion film, y lo definen como "producciones audiovisuales, a modo de cortometrajes, al servicio de una marca, caracterizadas por un estilo comunicativo en el que predomina la belleza y la estética extremadamente cuidada del mensaje -heredada de la fotografía de moda-, sobre el producto y/o la marca en sí mismos".

En las definiciones anteriores se reconoce el carácter estético y artístico de los fashion films y, como es propio de la industria cinematográfica, la participación notable de grandes directores, fotógrafos y actores famosos. De esta manera, como explican Caerols y De la Horra (2015, p. 354), "la belleza característica del fashion film es, en el fondo, una puesta para lograr que el espectador se acerque voluntariamente a su consumo como si se tratara de cualquier otro producto audiovisual". En este sentido, hay autores que identifican la presencia de la publicidad, pero de manera camuflada en las historias como Ramos-Serrano \& Pineda (2009, p. 730), quienes admiten que en los fashion films, como advertainment, "la publicidad se disfraza de contenido de entretenimiento". En esta línea, cabe destacar que el advertainment no deja de lado su condición publicitaria, refiriéndonos a la publicidad de la significación, con lo cual, como sustentan Pineda et al., (2013, p. 87), "el placement -de producto y/o marca- es aún un componente fundamental de dichas narraciones, de forma que el salto mercadotécnico hacia lo inmaterial no es absoluto". El estudio de Macías (2014) demuestra esta afirmación, quien ha analizado seis fashion films de la marca Dior ${ }^{3}$, y ha observado la implicación del producto integrado como un recurso narrativo en los guiones (figura 1).

"Lady Noire Affair" (2008), "Lady Rouge" (2010), "Lady Blue Shanghai" (2010), "Lady Grey London" (2011), "Lady Moscow" (2011) y Lady Dior (2011). 


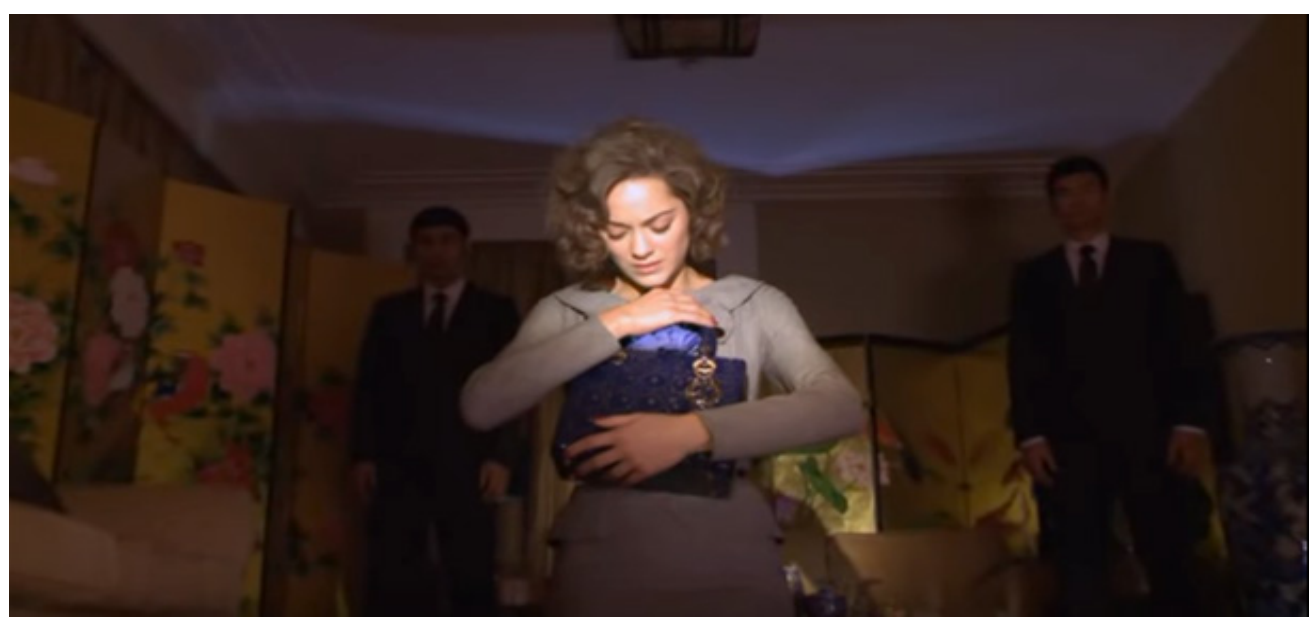

Figura 1. Escena del fashion film Lady Blue Shanghai (2010) de Dior, dirigido por David Lynch

En estos casos, es reconocible el plot placement, una versión activa del placement, característico del advertainment, que transforma al producto en protagonistas de una narración (Pineda et al., 2013). Así, la autora manifiesta que estos fashion films insertan los productos de las marcas de manera exagerada en las escenas y que, por lo tanto, revelan la índole comercial detrás de tales producciones audiovisuales. De esta manera, observamos que las marcas desarrollan los fashion films como advertainment, debido a la presencia explícita del producto y su protagonismo -actancial o de ayudante- en las historias, evidenciando su finalidad promocional.

Como se ha podido observar, la publicidad es connatural al fashion film. De hecho, si recurrimos a los antecedentes históricos de este tipo de contenido audiovisual, encontramos que su nacimiento se remonta hasta los inicios del cine, cuando el término "fashion film" fue por primera vez utilizado por la prensa en conexión con los newsreels ${ }^{4}$ de Pathé Frères Company en 1911, y cuando el deseo de extender la publicidad de marcas de moda hacia el ámbito audiovisual se hizo realidad con Georges Méliès ${ }^{5}$ (Uhlirova, 2013b). Dicho esto, como concluye Uhlirova (2013b, p. 153), "el fashion film, en general, siempre buscó conectar los intereses comerciales con el entretenimiento y el placer visual".

Así, hasta este punto es acertado reafirmar el advertainment como la naturaleza propia del fashion film en el contexto de los mensajes híbridos. Sin embargo, observamos una versión del fashion film que ha sido ignorada, y que verdaderamente responde a la naturaleza particular de la moda al prescindir del producto. Díaz y García (2016) definen los fashion films como una herramienta de branding que tiene como finalidad ofrecer al espectador una emoción o experiencia estética memorable que englobe los valores esenciales de una

El formato más popular de la época para promocionar los productos mediante una serie de consejos de moda (Uhlirova, 2013b).

5 Entre 1898 y 1900, Méliès, el pionero del féerie film, realizó el primer comercial para Mystère Corsers, una marca de corsés (Uhlirova, 2013b). 
marca. De este modo, mediante el entretenimiento liderado por historias coherentes con los valores de una marca, el consumidor deseará formar parte del universo exclusivo que se le presente. Así también, Buffo (2017, p. 296) sustenta que "los fashion films han desarrollado su propio lenguaje, creando imaginarios de marca y convirtiéndose en herramientas estratégicas para construir la identidad visual de las marcas de moda". En este sentido, el fashion film se erige como la forma de comunicación adecuada para narrativizar el mundo de las marcas de moda e integrar sus valores, excluyendo la promoción de productos como objetivo fundamental. Así, este contenido audiovisual, en vez otorgar protagonismo a los productos, debe concentrarse específicamente en contar una historia, dibujar una personalidad, y crear una atmosfera (Buffo, 2017).

Tras haber visto la naturaleza propia de las marcas de moda, y haber estudiado todas las categorías de branded content, sostenemos que los fashion films, además del advertainment, pueden ser considerados branded entertainment, o sea, como una técnica centrada en la transmisión de los valores de la marca a través de historias, omitiendo algún tipo de placement de los productos en la historia, dado que resulta innecesario, y porque además frustra la experiencia del espectador. Sin embargo, vemos que, en teoría, los fashion films no encajan del todo en la categoría branded entertainment, ya que en la industria cinematográfica -ropa, accesorios, calzado- es imposible evitar la presencia del producto, dado que los actores deben estar vestidos y, lógicamente, no lo estarán con los productos de la competencia. Además, como afirma Buffo (2016, p. 413), "la ropa es una herramienta importante para describir y mostrar las personalidades de los personajes de una película", con lo cual los productos de moda son una variable imprescindible en este caso. Cabe resaltar que los productos usados irremediablemente por los actores no deben tener un rol protagónico en la trama, sino simplemente funcionar como sus elementos de acompañamiento.

Un claro ejemplo de esto es el fashion film "High Tide" de la marca Dries Van Noten, ganador en la categoría de mejor fashion film a nivel nacional del Best National en la tercera edición del Madrid Fashion Film Festival, el cual cuenta la historia de un hombre que paralelamente se mira a sí mismo en vida y muerte rodeado de mujeres jóvenes. En palabras de su director Moya (2015), la historia "intenta ser una parábola del viaje de purificación al que se somete el alma cuando se separa del cuerpo ly] (...) describir un proceso de regeneración espiritual a la vez sofisticado y exhaustivo".

A efectos de guion, el fashion film "High Tide" presenta un planteamiento que inmediatamente es capaz de atrapar la atención del espectador, ya que se trata de la comprobación de la muerte del propio protagonista, quien se ve a sí mismo en ese trance. La tensión dramática se maneja sutilmente gracias al constante juego metafórico respecto a la vida y a la muerte, y al uso de la música in crescendo. El personaje evoluciona cuando reconoce su propia purificación y esto se aprecia en el fashion film con la aparición de su yo-niño, distinto a su yo-adulto, que hacia el final del video ya aparece caminando descalzo sobre las rocas junto al mar, como formando parte de la naturaleza misma. 


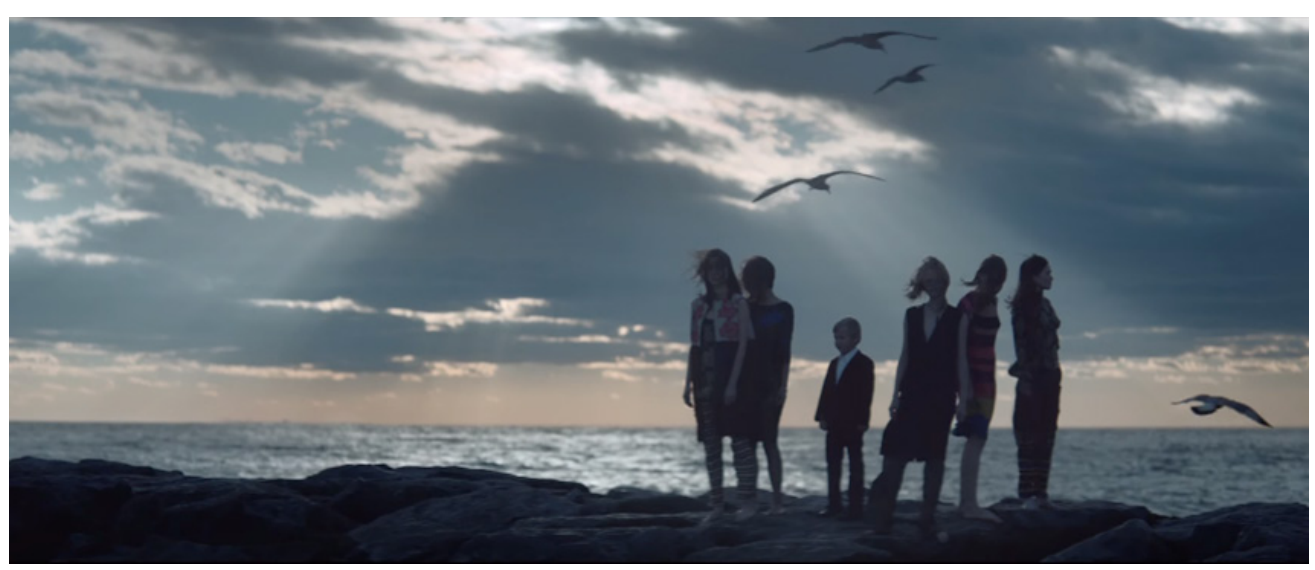

Figura 2. Escena del fashion film High Tide (2015) de Dries Van Noten, dirigido por Albert Moya.

Efectivamente, en este fashion film se pueden visualizar los productos de Dries Van Noten sobre los personajes, pero no percibirlos como objetos promocionales, ya que en ningún momento tienen un papel protagónico en la trama. De este modo, la concentración en la narrativa implica el cambio de la visión de consumidor a espectador (Khan, 2012). Dicho esto, determinamos que el branded entertainment aplicado en la industria de la moda es muy particular.

Después de un recorrido por los distintos mensajes híbridos, sostenemos que el fashion film, concebido como branded entertainment, es una herramienta que permite mostrar el mundo imaginario y exclusivo propio de cada marca de moda de lujo mediante la narración de historias vinculadas a sus valores, ya que esta técnica busca la concentración total del espectador en la historia, más no en los productos. En este sentido, las historias en los fashion films no son excusas para introducir nuevos productos, sino que son la representación de la sustancia de las marcas de moda de lujo. De este modo, el fashion film, como este tipo de mensaje, se erige como la práctica por excelencia para comunicar la identidad de las marcas de moda de manera auténtica, sin interrupción alguna, a pesar de permitir la visualización del producto, pero no como un objeto en venta, sino como un adorno que acompaña a los protagonistas para embellecerlos en la historia. De hecho, el fashion film procura impregnar los valores de marca en los personajes, las ambientaciones, en definitiva, en el mismo guion.

\section{Conclusiones}

El cine y la publicidad han crecido juntos, en el sector de la moda, desde inicios del siglo XX y, en la actualidad, han logrado aterrizar en el ámbito de los mensajes híbridos con el advertainment. Así, debido a las nuevas tecnologías, medios, y sobre todo audiencias, el carácter del fashion film en la era digital ha evolucionado notablemente. En este sentido, además del advertainment, los fashion films han dado un paso más hasta responder a la categoría de branded entertainment, aunque, debido a las particularidades del sector de la moda, es necesario admitir la presencia irreparable de los productos en la historia, siempre 
y cuando estos no formen parte de la narrativa al ser expuestos de manera protagónica, sino que sean objetos de embellecimiento de los personajes. En este sentido, el fashion film, como branded entertainment, es una fusión de las propiedades del cine y la moda, esta última concebida no como la exhibición de una serie de productos novedosos, sino fundamentalmente como un fenómeno social, cuyo objetivo es describir un determinado grupo social mediante la presentación de un mundo imaginario integrado de valores de la marca.

De este modo, partiendo de que el branded entertainment permite la construcción de mundos imaginarios mediante los cuales las marcas pueden visualizar su identidad y significado, y que la moda es un medio de integración y un recurso de embellecimiento, es posible sostener que, si el espectador llega a vincularse emocionalmente con la historia contada en un fashion film, él querrá formar parte de aquel universo imaginario mostrado. Para lograrlo, querrá imitar a los personajes y, por ende, se vestirá como ellos. Por ello, el fashion film se erige como una herramienta particular en las estrategias del marketing de moda del sector de lujo que, gracias a su doble naturaleza, puede desempeñarse bajo dos distintos objetivos de comunicación: el protagonismo del producto promocional en la historia correspondiente al advertainment, y la relevancia de los valores de marca en la narrativa perteneciente al branded entertainment.

\section{Referencias}

Aguado, G. (2008). Branded content: más allá del product placement en la televisión digital : advertainment y licensing. Enlaces: Revista del CES Felipe II, 5(8). Recuperado de http://www.cesfelipesegundo.com/ revista/articulos2008/Art\%c3\%adculo\%20Guadalupe\%20Aguado_corregido.pdf

Buffo, S. (2016). Fashion Films and Net-aesthetics. Journalism and Mass Communication, 6(7), 409-419. doi: 10.17265/2160-6579/2016.07.005

Buffo, S. (2017). Brand Narration and Fashion Films. Journalism and Mass Communication, 7(6), 292-304. doi: 10.17265/2160-6579/2017.06.002

Caballero, J. (2014). Branded content en las marcas de lujo (Trabajo de fin de grado). Universidad de Sevilla, España. Recuperado de https://goo.gl/6Cdvsa

Caerols, R. y De la Horra, Y. (2015). Fórmulas creativas en la publicidad de moda. Prisma Social: Revista de Investigación Social, (14), 336-378.

Caro, A. (1994). La publicidad que vivimos. Madrid: Celeste Eresma.

Castelló-Martínez, A. y Del Pino-Romero, C. (2014). Análisis del branded content en televisión: estudio de casos españoles. Revista de Comunicación de La SEECI, 18(34), 134-149. Recuperado de https:// goo.gl/fP8k28

Content Marketing Institute. (s. f.). What Is Content Marketing? Useful content should be at the core of your marketing. Recuperado de https://goo.gl/EKLck2

Cordero, J. (2015a). El coste alternativo en la valoración de los intangibles: caso práctico en comunicación. Revista de Contabilidad y Dirección, 21, 167-182. Recuperado de https://bit.ly/31 DoGmY

Cordero, J. (2015b). El emplazamiento de producto en la comunicación pulicitaria audiovisual (Tesis doctoral). Universidad Nacional de Educación a Distancia., España. Recuperado de https://goo.gl/4J4qr8 
De Aguilera, J., Baños, M. y Ramírez, F. (2016). Los mensajes híbridos en el marketing postmoderno: una propuesta de taxonomía. Revista ICONO14, 14(1), 26. doi: 10.7195/ri14.v14i1.890

De Aguilera-Moyano, J., Baños-González, M. y Ramírez-Perdiguero, J. (2015). Branded entertainment: los contenidos de entretenimiento como herramienta de comunicación de marketing. Un estudio de su situación actual en España. Revista Latina de Comunicacion Social, 70, 519-538. doi: 10.4185/ RLCS-2015-1057

Del Olmo, J. (2005). Marketing de la moda. Madrid: Ediciones Internacionales Universitarias.

Del Pino-Romero, C. y Castelló-Martínez, A. (2015). La comunicación publicitaria se pone de moda: branded content y fashion films. Revista Mediterránea de Comunicación, 6(1), 105-128. doi: 10.14198/ MEDCOM2015.6.1.07

Del Río, J. y Kaufmann, J. (2014). Revisión teórica de la agencia publicitaria en la cultura digital. AdComunica, (8), 57-72. doi: 10.6035/2174-0992.2014.8.5

Díaz, P. y García, L. (2016). Fashion films as a new communication format to build fashion brands. Communication y Society, 29(2), 45-61. doi: 10.15581/003.29.2.45-61

Eguizábal, R. (2007). Teoría de la publicidad. Madrid: Cátedra.

Formoso, M., Sanjuán, A. y Martínez, S. (2016). Branded content versus product placement: visibilidad , recuerdo y percepción del consumidor. Revista Pensar la Publicidad, 10, 13-25. doi: 10.5209/ PEPU.53771

Franck, R. (1990). La moda al descubierto. Barcelona: Costura-3 Ingenieros.

García, V., Sánchez, N. y Torrano, J. (2014). Actitud hacia el product placement en los videojuegos para móviles. Anuario de Jóvenes Investigadores, (7), 81-83. Recuperado de https://goo.gl/NMYnHh

Garrido, P. y Fernández, P. (2014). Branded content y Storytelling: la distancia más corta entre dos personas es una historia. En P. Fernández y S. Liberal (Eds.), Últimos estudios sobre publicidad: de "Las Meninas" a los tuits (pp. 35-48). Madrid: Fragua.

Gómez, A., Calvo, J. y Otero, C. (2013). Marketing y moda. Madrid: Editorial Académica Español.

Guerrero, D. (2015). Cómic y Brand Placement: análisis de la presencia y estrategia de las marcas comerciales en el cómic literario y cinematográfico (Tesis doctoral). Universidad de Málaga, España. Recuperado de https://goo.gl/bgqJWa

Hallahan, K. (2014). Publicity Under Siege: A Critique of Content Marketing, Brand Journalism, Native Advertising and Promoted User Endorsements As Challenges to Professional Practice and Transparency. En Y. Grace Ji, J. Helen Liu y Z. Cathy Li (Eds.), En 17th International Public Relations Research Conference (pp. 391-437). Coral Gables, Florida. doi: 10.1007/s13520-015-0046-y

Herrero, M. (2004). Fascinación a la carta. En M. Codina y M. Herrero (Eds.) Mirando la moda: once reflexiones (pp. 23-34). Madrid: Ediciones Internacionales Universitarias.

Kapferer, J.-N. y Valette-Florence, P. (2016). Beyond rarity: the paths of luxury desire. How luxury brands grow yet remain desirable. Journal of Product y Brand Management, 25(2), 120-133. doi: 10.1108/ JPBM-09-2015-0988

Kawamura, Y. (2005). Fashion-ology: an introduction to fashion studies. Nueva York: Berg.

Khan, N. (2012). Cutting the fashion body: Why the fashion image is no longer still. Fashion Theory, 16(2), 235-250. doi: 10.2752/175174112X13274987924177 
Khalbous, S., Vianelli, D., Domanski, T., Dianoux, C. y Maazoul, M. (2013). Attitudes toward Product Placement: A Cross-Cultural Analysis in Tunisia, France, Italy, and Poland. International Journal of Marketing Studies, 5(2), 138-153. doi: 10.5539/ijms.v5n2p138

Kumar, D. y Raju, K. (2013). The role of advertising in consumer decision making. IOSR Journal of Business and Management, 14(4), 37-45. Recuperado de https://bit.ly/2TZzpr]

Lehu, J. (2007). Branded entertainment: product placement and brand strategy in the entertainment business. London: Kogan Page.

Lieb, R. (2011). Content Marketing: Think Like a Publisher - How to Use Content to Market Online and in Social Media. Indianapolis: Que Publishing.

Macías, G. (2014). La integración de los valores de marca en los fashion films a través del guión : el caso Lady Dior (Trabajo fin de máster). Universidad de Sevilla, España. doi: 10.13140/2.1.1357.3440

Martín, M. (2008). El consumo de moda. «mposible» es un término desconocido para la moda. In Moda, Comunicación y Sociedad (Grupo de A, pp. 89-116). Sevilla: Comunicación social ediciones y publicaciones.

Martínez, J. (201 1). Hacia una taxonomía del advertainment. En I, Bort Gual, S. García Catalán y M. Martín Nuñez. (Eds.), Actas del IV Congreso Internacional sobre Análisis Fílmico: nuevas tendencias e hibridaciones de los discursos audiovisuales en la cultura digital contemporánea (pp. 549-562). Castellón: Ediciones de las Ciencias Sociales de Madrid. doi:10.6035/978-84-87510-57-1.2011.46

Martínez, E. y Vázquez, A. (2006). Marketing de la moda. España: Pirámide.

Méndiz, A. (2000). Nuevas formas publicitarias: patrocinio, product placement, publicidad en internet. Málaga: Servicio de Publicaciones Universidades de Málaga.

Michaud, Y. (2015). El nuevo lujo: experiencias, arrogancia, autenticidad. Madrid: Taurus.

Morgado, M. (2008). Del product placement no se escapa nadie. Fòrum de Recerca, (13), 388-396.

Moya, A. (2015). High Tide. Madridfff. Recuperado de https://bit.ly/2IRPkjz

Ortega, E. (1997). La comunicación publicitaria. Madrid: Pirámide.

Patrutiu, L. (2015). Content marketing: the fundamental tool of digital marketing. Bulletin of the Transilvania University of Brasov, 8(57), 111-118. Recuperado de https://goo.gl/jYv2qs

Pérez, J. , Navarrete, J. y Gómez, F. (2014). El product placement en el videoclip: análisis de contenido del emplazamiento de marca en los vídeos musicales preferidos por los usuarios de YouTube. Doxa Comunicación: Revista Interdisciplinar de Estudios de Comunicación y Ciencias Sociales, (18), 83-104. Recuperado de https://goo.gl/Ll pvo9

Pineda, A. (2009). Branded content antes del branded content: la modelación cultural porpagandística como forma de propaganda encubierta. Ámbitos, (18), 117-134. Recuperado de https://goo.gl/zCdEaw

Pineda, A., De Algaba Chicano, C. y Hernández-Santaolalla, V. (2013). La ficción como publicidad: análisis semiótico-narrativo del corporate advertainment. Área Abierta, 34(3), 67, 68-90. doi:10.5209/ rev_ARAB.2013.v34.n3.43354

Pulizzi, J. (2012). Six Useful Content Marketing Definitions. Recuperado de https://goo.gl/c76tSB

Rahim, K. y Clemens, B. (2012). Organizational Goals and Performance Measurement Criteria for Content Marketing. Journal of Communication and Computer, 9(8), 896-904. 
Ramos-Serrano, M. (2006). Cuando la marca ofrece entretenimiento: aproximación al concepto de advertainment. Questiones Publicitarias, 1(1 1), 33-49. Recuperado de https://goo.gl/FybRjj

Ramos-Serrano, M. y Macías-Muñoz, G. (2016). Branded content, branded entertainment y advertainment: definiciones y taxonomías. Actas del V Congreso Iberoamericano de Comunicación: Comunicación, Cultura y Cooperación (pp. 354-369). AE-IC. Recuperado de http://www.madrid2016.org/\#

Ramos-Serrano, M., y Pineda, A. (2009). El advertainment y las transformaciones de los formatos en el ámbito publicitario: el caso de los fashion films. Trípodos, 2009 (2), 729-737. Recuperado de https://bit.ly/2x209Ks

Rancati, E. y Gordini, N. (2014). Content Marketing Metrics: Theoretical Aspects and Empirical Evidence. European Scientific Journal, 10(34), 92-104. Recuperado de https://goo.gl/Hd9FP6

Redondo, I. y Bernal, J. (2015). Product placement: una revisión teórico-práctica de sus capacidades y limitaciones. Interciencia, 40(12), 827-833. Recuperado de https://goo.gl/Kyn31F

Regueira, F. (2012). El contenido como herramienta eficaz de comunicación de marca: análisis teórico y empírico (Tesis doctoral). Universidad Rey Juan Carlos, Madrid, España. Recuperado de https://goo.gl/or79il

Rodríguez, T. y Baños, M. (2013). El recuerdo del product placement en el espectador y su relación con la estructura dramática. Estudio de caso en el cine de Alex de la Iglesia. Communication y Society, 26(2), 147-175. Recuperado de https://goo.gl/ptvNFe

Rodríguez, A. (2012). La publicidad en el universo diegético del cine: análisis de cómo las marcas y los productos se integran a las tramas de las películas. Iconofacto, 8(11), 1-8. Recuperado de https:// goo.gl/MdJZMr

Sánchez, A. (1999). Moda: entre la ética y la estética. Madrid: Palabra.

Segarra-Saavedra, J. (2016). Panorama español de las webseries publicitarias. Aproximación transmediática desde la perspectiva del branded content (Tesis doctoral). Universidad de Alicante, España.

Segarra-Saavedra, J. e Hidalgo-Marí, T. (2013). Brand content y ficción televisiva. El caso de "I + B, Ir más a los bares" de Coca Cola. En V Congreso Internacional Latina de Comunicación (pp. 1-39). Universidad de La Laguna. Recuperado de https://goo.gl/Wr29qS

Segarra-Saavedra, J., Hidalgo-Marí, T. y Tur-Viñes, V. (2016). Branded webseries: acciones estratégicas del anunciante basadas en la ficción on line corporativa y el marketing de contenidos. Index.Comunicación, 6(2), 259-284. Recuperado de https://goo.gl/h1Sk4p

Semprini, A. (1995). El marketing de la marca: una aproximación semiótica. Barcelona: Paidós.

Suárez, B. (2012). La publicidad, el lenguaje del consumo: redefinición y prospectiva. Cuadernos del Centro de Estudios en Diseño y Comunicación. Ensayos, (39), 29-38. Recuperado de https://goo.gl/GKstt6

Uhlirova, M. (2013a). The Fashion-Film effect. In Djurdja Barlet, Shaun Cole and Agnes Rocamora (Eds.), Fashion Media: Interogating Words and Images (pp. 118-129). Oxford: Berg.

Uhlirova, M. (2013b). 100 years of the Fashion Film: Frameworks and histories. Fashion Theory, 17(2), 137-158.doi: 10.2752/175174113X13541091797562

Wells, W., Burnett, J. y Moriarty, S. (1996). Publicidad: principios y prácticas. Ciudad de Mexico: PrenticeHall Hispanoamericana. 